\title{
Theoretical study of the process of interaction of particles of weed impurities in the flow of raw cotton with the working bodies of the cleaning machine
}

\begin{abstract}
The article proposes to use the Euler equations to describe the motion of a stationary flow in the cleaning zones, which allows determining the laws of pressure, density and velocity distribution along the arc of contact of a moving layer of raw cotton with a mesh surface in the process of impact impact with spikes on the pulp. It has been established that the pressure, densities and flow rates along the cleaning arc as a result of hammer spikes vary in steps, with a decrease in pressure and density and an increase in the flow velocity along this arc.
\end{abstract}

Keywords: equations, friction force, mass of raw cotton, ring drums, mesh surfaces, dry friction force, model, density, angular velocity, element motion, elastic element, mote model
Volume 6 Issue 4 - 2020

\begin{abstract}
Gaybnazarov Egamnazar Eryigitovich,' Abdurakhmonov Akmalzhon Akbarovich ${ }^{2}$ 'Doctor tech. sciences, Department of Namangan Engineering and Technology Institute, Republic of Uzbekistan

${ }^{2}$ Lecturer, Namangan Institute of Engineering and Technology Republic of Uzbekistan
\end{abstract}

Correspondence: Abdurakhmonov Akmalzhon Akbarovich, Lecturer, Department of Namangan Engineering and Technology Institute, Namangan city, Republic of Uzbekistan,

Tel +998936773659, Email akm0 I0I I 97akm@gmail.com

\section{Introduction}

In world practice, large-scale research is being carried out to improve the technique and technology for processing cotton with high contamination, especially machine harvesting. In this area, the development of theoretical foundations of the technology for cleaning from weeds, the development of mathematical models that contribute to the establishment of optimal cleaning modes without negative impact on the initial quality indicators of cotton, the development of efficient and resource-saving devices, an effective technology for preparing raw cotton for the cleaning process, optimization of modes and parameters machines are becoming increasingly important.

In this article, the study of the possibility and preference of cleaning cotton before rioting, the development of an effective technology for preparing raw cotton for storage, based on this method, as well as the effect of this method on the release of trash from the cotton composition and on the initial quality indicators of cotton fiber is relevant.

The scientific significance of the research results is explained by the fact that in the process of research, the regularities of the movement of cotton in the working chamber of the purifier were revealed, the process parameters were optimized by means of mathematical models of the interaction of cotton with the surface of the working organs, and regularities were revealed that express the influence of the process parameters on the cleaning efficiency. The theoretical significance of the research results is due to the fact that, as a result of the research carried out on the recommended technology for cleaning cotton, a new arrangement of peg drums, working surfaces helps to preserve the natural indicators of the quality of raw cotton, to increase the efficiency of cleaning cotton from weeds and improve product quality.

To describe the process of purification of a stationary flow of raw cotton from weeds, it is proposed to use the model of A.G. Sevostyanov. The regularity of the distribution of the amount of released impurities both in the areas between the pegs and between the sections of the cleaning zone was established and it was shown that the largest amount of released impurities is released in the areas between the first and third pegs, then its slight drop occurs in the areas between the next pegs. This circumstance should be taken into account when choosing the length of the contact zones of the raw material with the mesh surface.

The equation of motion of the raw cotton flow in each section is described by the Euler equation

$$
\rho_{j} v_{j} \frac{d v_{j}}{d \alpha}=-\frac{d p}{d \alpha}+\rho_{j} g R(\cos \alpha-f \sin \alpha)-f \rho_{j} v_{j}^{2}
$$

Where $\rho_{j}=\rho_{j}(\alpha), v_{j}=v_{j}(\alpha)$, and $p_{j}=p_{j}(\alpha)$ density, speed and pressure of raw cotton in sector $\alpha_{j-1} \leq \alpha \leq \alpha_{j}$

Equation (1) contains 3 unknowns: $p, \rho$ and $v$. To close it, we use the equation of state of a compressible medium, which establishes a relationship between pressure $p$ and density $\rho$ :

$$
\rho=\rho_{c}\left[1+A\left(p-p_{c}\right)\right]
$$

and the condition of conservation of mass for stationary motion of the flow

$$
\rho v S_{0}=Q_{0}
$$

Here $S_{0}=k_{0} L h$ is the cross-sectional area of the flow layer, $h$ is the thickness of the layer, $L$ is the length of the drum, $k_{0}$ is the coefficient characterizing the decrease in the area of contact of the raw material with the surfaces of the pegs. $Q_{0}$ is the productivity of the purifier, $\rho_{c} p_{c}$ is the density and pressure when the raw material enters the cleaning zone, $A$ is the constant characterizing the compressibility of the raw material. 
From dependencies (2) and (3) with $A<<1$ we determine the speed $v=v_{c}\left[1-A\left(p-p_{c}\right)\right](4)$

Under impact action with a splitter on the surface of the contact of a particle, the flow acquires the velocity $v_{c}=\beta v_{k}$, where $v_{k}$ is the linear speed of the splitting, where $\beta<1$ is the coefficient of speed reduction, determined empirically, in ${ }^{1}$ the average flow rate in the cleaning zone is taken $v_{\mathrm{cp}}=0.5 v_{k}$

Assuming $v=v_{c}$ in formula (4), we find the density of the raw material when the raw material enters the cleaning zone $\rho_{c}=\frac{Q_{0}}{S_{0} v_{c}}$

To determine the pressure $p_{c}$, we consider the known pressure $p_{0}$ the density of the raw material $\rho_{0}$ in the feed zone. Then putting $p=p_{0}$ and $\rho=\rho_{0}$ to formula (3), we find

$$
p_{c}=p_{0}-\left(\rho_{0} / \rho_{c}-1\right) / A
$$

From the requirement of the absence of separation of raw materials from the splitting surface it follows $p_{c}>0$, which means $\frac{\rho_{0}}{\rho_{c}}<1+p_{0} A$. On the other hand, the condition of rarefaction of raw $\rho_{c}$
materials in the cleaning zone $p_{c}<p_{0}$, which gives $\frac{\rho_{0}}{\rho}>1$, must be fulfilled. $\rho_{c}$

Thus, to implement the process of loosening the raw material without breaking contact with the splitter, it is necessary that the density ratio $\frac{\rho_{0}}{\rho_{c}}$ satisfies inequality $1<\frac{\rho_{0}}{\rho_{c}}<1+p_{0} A$.

The limitation on the pressure value $p_{0}$ (or the splitting speed) follows from the condition that there is no damage to the seeds during the impact interaction of the splitter with the raw material. If we denote by $P_{k}$ the limiting impact force at which the seeds are damaged, then assuming in formula (6) $p_{c}<P_{k} / S_{0}$, we obtain $p_{0}<P_{k} / S_{0}+\left(\rho_{0} / \rho_{c}-1\right) / A$

We introduce a new variable according to the formula $\alpha=s / R$ ( $\alpha$ is the central angle, $R$ is the radius of the drum). Taking into account (2) and (4), we write down the equation for pressure $p$.

$a \frac{d p}{d \alpha}=R \rho g(\sin \alpha-f \cos \alpha)\left[1+A\left(p-p_{c}\right)\right]-\overline{Q_{0}} f\left[1-A\left(p-p_{c}\right)\right]$

Equation (6) is reduced to the form:

$$
\begin{gathered}
\frac{d p}{d \alpha}=F_{1}(\alpha) p+F_{2}(\alpha) \\
\text { Where } F_{2}(\alpha)=\frac{A\left[R \rho_{0} g F_{1}(\alpha)+\bar{Q}_{0} f v_{0}\right]}{a}, \\
F_{4}(\alpha)=\frac{\left(1-A p_{c}\right) F_{1}(\alpha) R \rho_{0} g-\overline{Q_{0}} v_{0} f\left(1+p_{c} A\right)}{a} \\
F_{1}(\alpha)=\sin \alpha-f \cos \alpha, a=1-\overline{Q_{0}} v_{c} A, \overline{Q_{0}}=\frac{Q_{0}}{S_{0}} .
\end{gathered}
$$

The solution to equation (7) satisfying condition $p(0)=p_{c}$ is represented in quadratures $p=F_{3}(\alpha)\left[\frac{p_{0 c}}{F_{3}(0)}+\int_{0}^{\alpha} \frac{F_{4}(\alpha)}{F_{3}(\alpha)} d \alpha\right]$

where $F_{3}(\alpha)=\exp \left[\int F_{2}(\alpha) d \alpha\right]$.

We use formula (8) to determine the pressure $p$ in each section.

Let us consider the case when the contact of the flow of raw cotton in the first section with the mesh surface occurs in four sections $0<\alpha<\alpha_{0} \alpha_{0}<\alpha<2 \alpha_{0}, \quad 2 \alpha_{0} \leq \alpha<3 \alpha_{0}$ and $3 \alpha_{0} \leq \alpha<4 \alpha_{0}$

The solution at each section, taking into account the change in contact pressure according to the formula (8), in the presence of a blow with each peg, is written in the form:

$$
\begin{gathered}
p=p_{1}=F_{3}(\alpha)\left[\frac{p_{c}}{F_{3}(0)}+\int_{0}^{\alpha} \frac{F_{4}(\alpha)}{F_{3}(\alpha)} d \alpha\right] \text { at } 0<\alpha<\alpha_{0} \\
p=p_{2}=F_{3}(\alpha)\left[\frac{p_{1 c}}{F_{3}\left(\alpha_{0}\right)}+\int_{\alpha_{0}}^{\alpha} \frac{F_{4}(\alpha)}{F_{3}(\alpha)} d \alpha\right] \text { at } \alpha_{0}<\alpha<2 \alpha_{0} \\
p=p_{3}=F_{3}(\alpha)\left[\frac{p_{2 c}}{F_{3}\left(2 \alpha_{0}\right)}+\int_{2 \alpha_{0}}^{\alpha} \frac{F_{4}(\alpha)}{F_{3}(\alpha)} d \alpha\right] \text { at } 2 \alpha_{0}<\alpha<3 \alpha_{0} \\
p=p_{4}=F_{3}(\alpha)\left[\frac{p_{3 c}}{F_{3}\left(3 \alpha_{0}\right)}+\int_{3 \alpha_{0}}^{\alpha} \frac{F_{4}(\alpha)}{F_{3}(\alpha)} d \alpha\right] \text { at } 3 \alpha_{0}<\alpha<4 \alpha_{0}
\end{gathered}
$$

Similarly, for the second section, we have

$$
\begin{aligned}
& p=p_{5}=F_{3}(\alpha)\left[\frac{p_{4 c}}{F_{3}(0)}+\int_{0}^{\alpha} \frac{F_{4}(\alpha)}{F_{3}(\alpha)} d \alpha\right] \text { at } 0<\alpha<\alpha_{0} \\
& p=p_{6}=F_{3}(\alpha)\left[\frac{p_{5 c}}{F_{3}\left(\alpha_{0}\right)}+\int_{\alpha_{0}}^{\alpha} \frac{F_{4}(\alpha)}{F_{3}(\alpha)} d \alpha\right] \text { at } \alpha_{0}<\alpha<2 \alpha_{0} \\
& p=p_{7}=F_{3}(\alpha)\left[\frac{p_{6 c}}{F_{3}\left(2 \alpha_{0}\right)}+\int_{2 \alpha_{0}}^{\alpha} \frac{F_{4}(\alpha)}{F_{3}(\alpha)} d \alpha\right] \text { at } 2 \alpha_{0}<\alpha<3 \alpha_{0}
\end{aligned}
$$

$$
p=p_{8}=F_{3}(\alpha)\left[\frac{p_{7 c}}{F_{3}\left(3 \alpha_{0}\right)}+\int_{3 \alpha_{0}}^{\alpha} \frac{F_{4}(\alpha)}{F_{3}(\alpha)} d \alpha\right] \text { at } 3 \alpha_{0}<\alpha<4 \alpha_{0}
$$

Where

$$
\begin{gathered}
p_{1 c}=p_{1}\left(\alpha_{0}\right)-\left[\frac{\rho_{1}\left(\alpha_{0}\right)}{v_{c}}-1\right] / A, \\
p_{2 c}=p_{2}\left(2 \alpha_{0}\right)-\left[\frac{\rho_{2}\left(2 \alpha_{0}\right)}{v_{c}}-1\right] / A,
\end{gathered}
$$




$$
\begin{aligned}
& p_{3 c}=p_{3}\left(3 \alpha_{0}\right)-\left[\frac{\rho_{3}\left(3 \alpha_{0}\right)}{v_{c}}-1\right] / A, \\
& p_{5 c}=p_{5}\left(\alpha_{0}\right)-\left[\frac{\rho_{5}\left(\alpha_{0}\right)}{v_{c}}-1\right] / A, \\
& p_{5 c}=p_{5}\left(\alpha_{0}\right)-\left[\frac{\rho_{5}\left(\alpha_{0}\right)}{v_{c}}-1\right] / A, \\
& p_{6 c}=p_{6}\left(2 \alpha_{0}\right)-\left[\frac{\rho_{6}\left(2 \alpha_{0}\right)}{v_{c}}-1\right] / A, \\
& p_{7 c}=p_{7}\left(3 \alpha_{0}\right)-\left[\frac{\rho_{7}\left(3 \alpha_{0}\right)}{v_{c}}-1\right] / A
\end{aligned}
$$

For the calculation, the reduced coefficient of friction between the mesh and raw cotton was used according to the formula $f=f_{0}(1-n)$ , where $n=S / S_{0}, S_{0}$ is the area of the mesh occupied by open areas, $S_{0}$ is the total area of the mesh.

Let us consider the process of separating weed impurities from the composition of raw cotton when it moves along a mesh surface. Following [3], the relationship between the mass $m$ of the raw cotton entering the cleaning zone and its density $\rho$ is represented in the form

$$
\frac{d m}{m}=\lambda \frac{d \rho}{\rho}
$$

Where $\lambda=1 /(1+a), a>0$ is the proportionality coefficient.

I integrate the last equation that satisfies the conditions $m=m_{9}$ $m_{0}$ is the mass of raw cotton entering the zone between the first and the second splitter of the cleaning zone of raw cotton per unit time), $\rho=\rho_{c}$ with $\alpha=0$ for the cleaning zone between the first and second splitting, we get

$$
\frac{m_{1}}{m_{0}}=\left(\frac{\rho_{1}}{\rho_{c}}\right)^{\lambda}
$$

Taking into account dependence (3), we have

$$
\frac{m_{1}}{m_{0}}=\left[1+A\left(p_{1}-p_{c}\right]^{\lambda} \text { for } 0<\alpha<\alpha_{0}\right.
$$

The mass of the separated impurity referred to the mass $m_{0}$, between the first and second, second and third, third and fourth pegs and after the impact of the fourth peg is determined by the formula

$$
\begin{gathered}
\varepsilon_{1}=\frac{m_{0}-m_{1}}{m_{0}}=1-\left[1+A\left(p_{1}-p_{0 c}\right]^{\lambda} \text { at } 0<\alpha<\alpha_{0}\right. \\
\varepsilon_{2}=\varepsilon_{1}\left(\alpha_{0}\right)\left(\frac{\rho_{2}}{\rho_{c}}\right)^{\lambda}=\varepsilon_{1}\left(\alpha_{0}\right)\left[1+A\left(p_{2}-p_{1 c}\right)\right]^{\lambda} \text { at } \alpha_{0}<\alpha<2 \alpha_{0} \\
\varepsilon_{3}=\varepsilon_{2}\left(2 \alpha_{0}\right)\left[1+A\left(p_{3}-p_{2 c}\right]^{\lambda} \text { at } 2 \alpha_{0}<\alpha<3 \alpha_{0},\right. \\
\varepsilon_{4}=\varepsilon_{3}\left(3 \alpha_{0}\right)\left[1+A\left(p_{4}-p_{3 c}\right]^{\lambda} \text { at } 3 \alpha_{0}<\alpha<4 \alpha_{0}\right.
\end{gathered}
$$

Similarly, for the second cleaning zone, we have

$$
\begin{gathered}
\varepsilon_{5}=\varepsilon_{4}\left(4 \alpha_{0}\right)\left[1+A\left(p_{5}-p_{4 c}\right]^{\lambda} \text { at } 0<\alpha<\alpha_{0},\right. \\
\varepsilon_{6}=\varepsilon_{5}\left(5 \alpha_{0}\right)\left[1+A\left(p_{6}-p_{5 c}\right]^{\lambda} \text { at } \alpha_{0}<\alpha<2 \alpha_{0}\right. \\
\varepsilon_{7}=\varepsilon_{6}\left(2 \alpha_{0}\right)\left[1+A\left(p_{7}-p_{6 c}\right]^{\lambda} \text { at } 2 \alpha_{0}<\alpha<3 \alpha_{0},\right. \\
\varepsilon_{8}=\varepsilon_{7}\left(3 \alpha_{0}\right)\left[1+A\left(p_{8}-p_{7 c}\right]^{\lambda} \text { at } 3 \alpha_{0}<\alpha<4 \alpha_{0}\right.
\end{gathered}
$$

Where pressure $p_{i} \quad(i=1,2,3 \ldots 8)$ is determined using formulas (9)-(16). The total mass of the separated trash impurities (referred to the total mass of raw cotton on the surface of the mesh) from the two cleaning zones is presented as a sum

$$
M=\sum_{i=1}^{4} \int_{(i-1) \alpha_{0}}^{i \alpha_{0}} \varepsilon_{i} d \alpha+\sum_{i=1}^{4} \int_{(i-1) \alpha_{0}}^{i \alpha_{0}} \varepsilon_{4+i} d \alpha
$$

\section{Analysis of the results}

(Figures 1-3) shows the graphs of the distribution of the density, velocity and mass of the separated impurities (referred to the mass of raw cotton) along the arc of contact of the raw cotton with the mesh surface of the first cleaning zone at two values of the productivity of the cleaning machine.

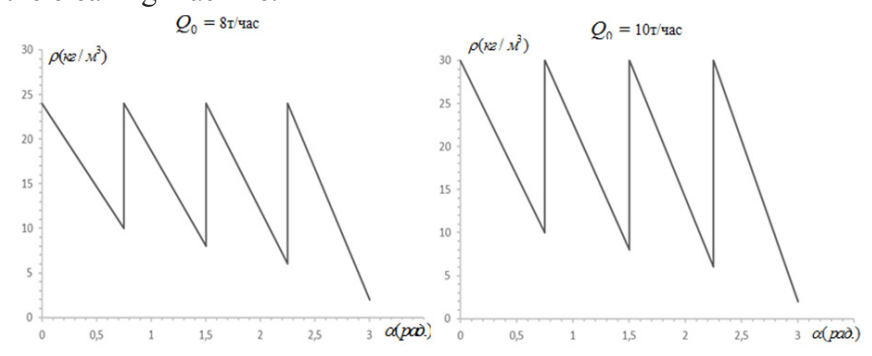

Figure I Density distribution $\rho\left(\mathrm{kg} / \mathrm{M}^{3}\right)$ of raw cotton in the area of the first cleaning section for two performance values $Q_{0}$. $Q_{0}=8 \mathrm{~T} / \mathrm{mac}$ $Q_{0}=10 \mathrm{~T} /$ чac $V\left(\kappa 2 / M^{3}\right)$ $v\left(x e / \mu^{3}\right)$

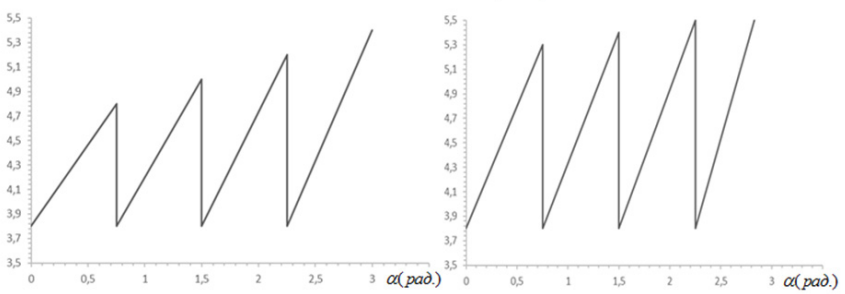

Figure 2 The distribution of the flow rate of raw cotton $v(\mathrm{M} / \mathrm{c})$ in the area of the first cleaning section for two performance values $Q_{0}$.
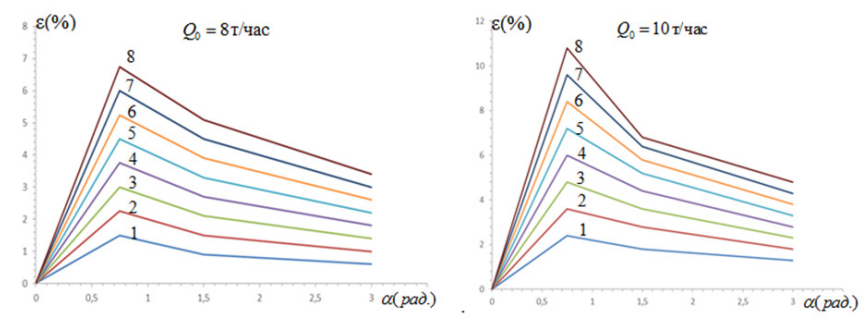

Figure 3 The mass distribution of the selected weed impurities (referred to the mass of raw cotton of raw) $\varepsilon$ (in percent) in the first section of the cleaning at two values of productivity $Q_{0}$ different values of parameter $\lambda$ $1-\lambda=0.06,2-\lambda=0.08,3-\lambda=0.1,4-\lambda=0.12,5-\lambda=0.14$ , $6-\lambda=0.16,7-\lambda=0.18,8-\lambda=0.2$. 
In calculations it is accepted: $R=0.2 \mathrm{M}, \omega=50 c^{-1}$ $h=0.018 \mathrm{M} ; h=0.018 \mathrm{M} ; L=1.7 \mathrm{M}, \alpha_{0}=45^{0}, \mathrm{k}_{0}=0.8$, $S_{0}=k_{0} h L=0.02448 \mathrm{~m}^{2}, \mathrm{f}=0.1, \rho_{0}=40 \mathrm{~kg} / \mathrm{m}^{3}, p_{0}=2500 \Pi \mathrm{a}$, $A=7 \cdot 10^{-4} 1 /$ Па From the analysis of the graphs, it follows that as a result of the impact of the pegs, the density and velocity in the sections of the flow layer at the impact sites change abruptly, while the density changes insignificantly during transitions to the sections between the pegs, an intensive increase in speed is observed, this is noticeable at high machine productivity (Figure 2).

The graphs of the distribution of the masses of trash impurities released from the flow (referred to the mass of the unrefined mass of raw cotton), shown in Figure 3, show that a high cleaning effect is observed in the area between the first and second splits, then there is a decrease in the mass of separated trash impurities, a significant impurities in the sections between the second and third headers are observed at high values of productivity $Q_{0}$. It can be seen that an increase in the value of parameter $\lambda$ leads to an increase in the mass of emitted trash. Based on the calculations, the total mass of trash impurities isolated from the cleaning zone was calculated.

Table 1 and Table 2 show the amounts of separated trash impurities in the areas between the pegs and their total weight (referred to the weight of raw cotton) at different values of parameter $\lambda$ and two values of productivity $\lambda$. From the analysis of the tabular data, it follows that the total mass of the released impurities can increase significantly at large values of the parameter $\lambda$. In this case, intensive release of trash impurities occurs in the areas between the first and second grates. ${ }^{1-9}$

Table I Values of the mass of separated trash impurities between the pegs and their total mass (attributed to the mass of raw cotton, in\%) in the first section of the cleaning zone at $Q_{0}=20 / 9 \mathrm{~kg} / \mathrm{c}$ and different values of the parameter $\lambda$

\begin{tabular}{lllllllllll}
\hline$\lambda$ & 0.06 & 0.08 & 0.1 & 0.12 & 0.14 & 0.16 & 0.18 & 0.2 & 0.2 & 0.24 \\
$0<\alpha \leq \alpha_{0}$ & 0.731 & 0.972 & 1.211 & 1.448 & 1.685 & 1.92 & 2.153 & 2.385 & 2.615 & 2.844 \\
$\alpha_{0}<\alpha \leq 2 \alpha_{0}$ & 1.438 & 1.901 & 2.356 & 2.803 & 3.242 & 3.674 & 4.098 & 4.515 & 4.925 & 5.328 \\
$2 \alpha_{0}<\alpha \leq 3 \alpha_{0}$ & 1.4 & 1.833 & 2.251 & 2.653 & 3.041 & 3.415 & 3.775 & 4.12 & 4.453 & 4.772 \\
$3 \alpha_{0}<\alpha \leq 4 \alpha_{0}$ & 1.369 & 1.78 & 2.169 & 2.239 & 2.888 & 3.219 & 3.532 & 3.828 & 4.107 & 4.369 \\
& & & & & & & & & & \\
$M_{k}=\sum_{i=1}^{4} M_{i k}(\%)$ & 4.937 & 6.485 & 7.987 & 9.444 & 11.29 & 12.23 & 13.56 & 14.85 & 16.1 & 17.31 \\
$k=1,2,3 \ldots 10$ & & & & & & & & & &
\end{tabular}

Table 2 Values of the mass of separated trash impurities between the pegs and their total mass (referred to the mass of raw cotton, in \%) in the first section of the cleaning zone at (I) and different values of the parameter (2)

\begin{tabular}{|c|c|c|c|c|c|c|c|c|c|c|}
\hline$\lambda$ & 0.06 & 0.08 & 0.1 & 0.12 & 0.14 & 0.16 & 0.18 & 0.2 & 0.2 & 0.24 \\
\hline $0<\alpha \leq \alpha_{0}$ & 0.731 & 0.972 & 1.211 & 1.448 & 1.685 & 1.92 & 2.153 & 2.385 & 2.615 & 2.844 \\
\hline$\alpha_{0}<\alpha \leq 2 \alpha_{0}$ & 1.438 & 1.901 & 2.356 & 2.803 & 3.242 & 3.674 & 4.098 & 4.515 & 4.925 & 5.328 \\
\hline $2 \alpha_{0}<\alpha \leq 3 \alpha_{0}$ & I. 4 & 1.833 & 2.251 & 2.653 & 3.041 & 3.415 & 3.775 & 4.12 & 4.453 & 4.772 \\
\hline $3 \alpha_{0}<\alpha \leq 4 \alpha_{0}$ & 1.369 & 1.78 & 2.169 & 2.239 & 2.888 & 3.219 & 3.532 & 3.828 & 4.107 & 4.369 \\
\hline$M_{k}=\sum_{i=1}^{4} M_{i k}(\%)$ & 4.937 & 6.485 & 7.987 & 9.444 & 11.29 & 12.23 & 13.56 & 14.85 & 16.1 & $|7.3|$ \\
\hline$k=1,2,3 \ldots 10$ & & & & & & & & & & \\
\hline
\end{tabular}

\section{Conclusion}

It is proposed to use the Euler equations to describe the motion of a stationary flow in the cleaning zones, which makes it possible to determine the laws of distribution of pressure, density and velocity along the arc of contact of a moving layer of raw cotton with a mesh surface in the process of impact by pegs on the fibrous mass. It has been established that the pressure, density and flow velocity along the cleaning arc as a result of blows with coke changes abruptly, with a decrease in pressure and density and an increase in the flow velocity along this arc. This indicates a process of significant loosening of the flow during the transition from the cleaning section to the second and there is a slight change in their values in other cleaning sections. It is proposed to use the model of A.G. Sevostyanov to describe the process of cleaning raw cotton from weeds. Equations have been drawn up to determine the amount of separated impurities both in the areas between the pegs and between the sections of the cleaning zone. It has been established that the largest amount of emitted impurities is released in the areas between the first and third pegs, then it falls slightly in the areas between the next pegs. This circumstance should be taken into account when choosing the length of the contact zones of the raw material with the mesh surface. 


\section{Acknowledgments}

None.

\section{Funding}

None.

\section{Conflicts of interest}

The authors have no conflicts of interest regarding the publication of this paper.

\section{References}

1. Omonov FB. Cotton processing directory. Toshkent; 2008.

2. Lebedev DA, Korabelnikov AR, Korabelnikov RV. Mechanism for removing weed impurities in the cleaning process. Textile industry technology; 2009.

3. Sevostyanov AG. Methods and tools for the study of mechanical and technological processes in the textile industry. Moscow Light Industry; 1980.
4. Burnashev RZ. Theoretical foundations of the technology of cleaning raw cotton. Tashkent; 1983.

5. Korabelnikov AR, Korabelnikov RV, Lebedev DA. Theoretical aspects of the mechanism of separation of weed particles from fibrous material when layers are shifted (unloading). Journal Technology of the textile industry. 2010;8(329).

6. Gaibnazarov EE, Obidov A, Kamolidinov M. Theoretical study of the process of cleaning raw cotton from large and small trash impurities under mechanical influence. Scientific and technical journal Problems of Mechanics. 2016;2:38-43.

7. Gaybnazarov EE, Xojiev MT, Isaev SH, et al. Modeling the process of separation of small contaminants into the stream of raw cotton moving in the area of treatment. International Journal of Advanced Research in Science, Engineering and Technology. 2018;11:74-78.

8. Gaibnazarov E, Khozhiev MT, Isaev Sh, et al. Analysis of the dynamic model of the interaction of trash impurities and theoretical study of the mixing of litter in raw cotton when cleaning it from trash. Journal Universum: technical sciences Moscow. 2018;4:23-26.

9. Gaybnazarov EE, Mardonov B, Azizov Sh. Theoretical study of movement trash in cotton lobules in cleaner small and large trash. Trends in Textile \& Flash Design. 2018;1(1). 